\title{
Comparison of Feed Forward Network and Radial Basis Function for Detecting and Recognition of License Plate
}

\author{
Pragya Bagwari \\ PG Scholar- GEU Dun
}

\author{
Bhavya Saxena \\ PG Scholar-GEU Dun \\ Sumit Pundir \\ PG Scholar-GEU Dun
}

\author{
Swapnil Bagwari \\ PG Scholar-LPUPhagwara
}

\begin{abstract}
One of the most important topics of intelligent transportation system is the License Plate Recognition (LPR). LPR systems have many potential applications in intelligent traffic systems, such as the payment of parking fee, highway toll fee, traffic data collection, traffic monitoring systems, traffic law enforcement, security control of restricted areas and so on. LPR was developed to identify vehicles by the contents of their license plates. The LPR system consists of three major modules: license plate extraction, segmentation and recognition of individual characters. This paper presents a study of applying the neural network approach for character image recognition. The new approach is tested on 400 samples of extracted license plate images captured in outdoor environment. The result yield $99.2 \%$ recognition accuracy, the method takes 1.6 seconds to perform the car plate recognition from vehicle's image. In order to decrease problems such as low quality and low contrast in the vehicle images, image recognition is done by the two different methods first is feed forward method and another is Radial Basis function and the best one is selected. The algorithm based on neural network which can quickly and correctly detect the region of license plate and the license plate detecting rate of success is $99.2 \%$.
\end{abstract}

\section{Keywords:}

Connected Components, Feed Forward Neural Network, Radial Basis Function

\section{INRODUCTION}

License plate number are being used to uniquely identify a vehicle. An automated system is developed in MATLAB which extracts the number plate and recognize alphanumeric characters. Techniques used for number plate extraction and recognition is feed forward neural network and radial basis function. During past few years, license plate recognition have been widely used as a core technology for security or traffic applications such as in traffic surveillance, parking lot access control and information management.

The complexity of number plate recognition work varies throughout the work. In India this task becomes much difficult due to variation in plate model. For the standard number plate, NPR system is easier to read and recognize.

\section{LITERATURE SURVEY}

Muhammad H. Dashtban, Zahra Dashtban et al. [1] proposed the algorithm that has three main parts, first is localization, second is segmentation and third is recognition. First the noise from the image is eliminated and then the rgb image is transformed to gray scale image. Then vertical sobel filter is used to detect the edges in image. Then 8 -connectives are determined which contain the desired regions. The various geometric features of regions are calculated like aspect ratio, area, range of length and width, region intensity. After the plate localization 2-d median filter is applied to remove some unwanted noise then contrast enhancement is done using histogram equalization. Then canny line detection algorithm and hough transform are used detect the longest line in image. Then each individual character is segmented. Then segmented block is prepared for neural networks using erosion and dilation. Then multi neuron perceptron are used to recognize the characters.

In [2] A.Broumandnia, M.Fathy, proposed a method is developed to scan a vehicle image with $\mathrm{N}$ row distance and count the existent edges. If the number of the edges is greater than a threshold value, this manifests the

presence of a plate. If in the first scanning process the plate is not found, then the algorithm is repeated reducing the threshold for counting edges. The method features very fast execution times as it scans some rows of the image. Nonetheless, this method is extremely simple to locate license plates in several scenarios and moreover it is not size or distance independent.

Anish Lazrus, Siddhartha Choubey, Sinha G.R. et al. [3] presents a powerful method of license plate location, segmentation and recognition of the characters in licence plate. The images of vehicles are converted into gray-scale images. To remove noise present in the plates wiener2 filter is used and edges are found using Sobel. Then bwlabel commond is used to calculate the connected component. Finally, single character is extracted from the licence plate. The proposed method achieved accuracy of $96 \%$ by optimizing various parameters.

H.J. Kim et. al [4] proposed a method of extracting plate region based on colour image segmentation. In this study, the proposed approach is based on four different methods extraction of plate region, segmentation of plate characters and recognition of characters.

Pranathi,A.Srisaila[6] proposed Automatic Number Plate Recognition which is a mass surveillance system that captures the image of vehicles and recognizes their license number. ANPR can be assisted in the detection of stolen vehicles. The detection of stolen vehicles can be done in an efficient manner by using the ANPR systems located in the highways. This paper presents a recognition method in which the vehicle plate image is obtained by the digital cameras and the image is processed to get the number plate information. A rear image of a vehicle is captured and processed using various algorithms. In this context, the number plate area is localized using a novel feature-based number plate algorithms i.e., Edge Finding Method and Window Filtering Method for the better development of the number plate detection system.

Mahood Ashoori-Lalimi[8] proposed an efficient method for licence plate localization in the images with many situations and complex background. In this they used edge density as pre 
processing for image enhancement[7]. The steps are in figure1.

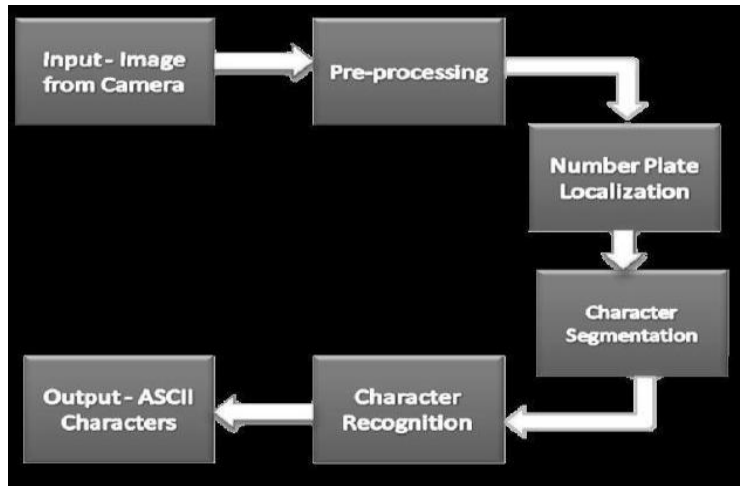

Fig1 : Steps used in vehicle license plate recognition system

\section{SYSTEM OVERVIEW}

A. Structure of the LPR system

The method proposed in this paper is designed for detection and recognization of license plates of vehicles automatically. Input of the system is the image of a vehicle captured by a camera. There are three modules first is extraction second is segmentation and third and last is recognition. Extraction part extracts the license plate from the image of a car. Segmentation part separates the characters individually. And finally recognition part recognizes the characters of license plate giving the result as the plate number. This is done by two methods first is by feed forward method and another is radial basis function and also the comparison is done to show which one is better.

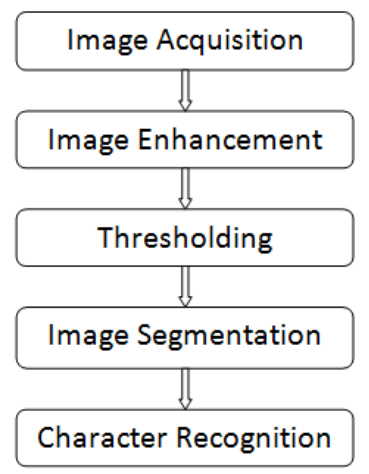

\section{Fig2 : Steps for the recognition of the license plate}

\section{B. Plate region extraction}

Plate region extraction is the first stage in this algorithm. Image captured from the camera is first converted to the gray scale image and then in binary image consisting of only 1's and 0 's (only black and white). Binary image is quantized into two values representing 0 and 1 or in pixel values of 0 and 255 representing the colour black and white. It is useful as the information we need, can be obtained from the silhouette of the object. The application is text interpreting and identifies the object orientations. Binary images are obtained by converting the input image into gray scale format, then by converting the gray scale image to binary image by thresholding. The image is made up of a matrix squares which is called pixel. Each pixel in the image has a brightness value which is known as gray level. The pixel of gray level above the threshold will be set to 1 or equal to 255 (white) and the rest will be set to 0 (black). We will get white object with black background or vice versa.

\section{Image Segmentation}

Image segmentation plays a vital and critical step that lead to the analysis of the processed image data. In order to extract and analyzed the object characteristic, the process need to partition the image into different parts that will have a strong correlation with the objects. Segmentation process can be categorized into several parts. Firstly is the global knowledge of an image. Secondly is the boundary-based segmentation. The process uses the edge detection to obtain the region contours and the objects will construct from the obtain contours. Lastly will be the edge-based segmentation.

The first step is to filter the image for enhancing and removing the unwanted spots. Then edge-based method is applied to the image for separating the characters from each other if the characters are close to each other. After this operation, horizontal and vertical operation are applied for finding the character regions. The next step is to cut the plate characters. It is done by finding the starting and end points of characters in horizontal direction and at last we get the segmented characters.

\section{Character Recognition}

Here Character Recognition is done by two methods first is feed forward neural network and another is Radial Basis Function.

1) Feed Forward neural network: A feed forward neural network is also called an artificial neural network in which connections between the units do not form a directed cycle. In this neural network, the information moves in only one direction, start from the input nodes, then through the hidden nodes and at last to the output nodes. There are no cycles or loops in the network.

(a)Experimental Result By Feed Forward Network: Experiments have been performed to test the proposed system and to measure the accuracy of the system. The system is designed in MATLAB 3 for recognition of Turkish license plates and some other images by camera. The images for the input to the system are coloured images. The test images were taken under various illumination conditions by the camera. The results of the tests are given by Table I.

Table I Experimental Result By Feed Forward Neural Network

\begin{tabular}{|l|l|l|}
\hline $\begin{array}{l}\text { Units of LPR } \\
\text { System }\end{array}$ & $\begin{array}{l}\text { Number of } \\
\text { Accuracy }\end{array}$ & $\begin{array}{l}\text { Percentage of } \\
\text { Accuracy }\end{array}$ \\
\hline $\begin{array}{l}\text { Extraction of } \\
\text { plate region }\end{array}$ & $231 / 250$ & $92.1 \%$ \\
\hline Segmentation & $221 / 250$ & $88.4 \%$ \\
\hline $\begin{array}{l}\text { Recognition of } \\
\text { Characters }\end{array}$ & $242 / 250$ & $96.5 \%$ \\
\hline
\end{tabular}

It is shown that accuracy for the extraction of plate region is $92.1 \%$, for the segmentation of characters is $88.4 \%$ and the percentage of accuracy of the recognition unit is $96.5 \%$. The overall system performance can be defined as the product of all units accuracy rates (Extraction of license plate region, segmentation of characters and recognition of characters). 
2) Radial Basis Function: A radial basis function network is also called an artificial neural that uses radial basis functions as activation functions. RBF networks have fixed three layer architecture which consists of first the input layer, second is the hidden layer and last one is output layer. The input layer provides network inputs, the hidden layer remaps the input data in order to make them linearly separable the output layer does linear separation.RBF Networks take a slightly different approach. Their main features are:

i) They are two-layer feed-forward networks.

ii) The hidden nodes implement a set of radial basis functions (e.g. Gaussian functions).

iii) The output nodes implement linear summation functions as in an MLP.

iv) The network training is divided into two stages: First the "weights" from the input to hidden layer are determined, and then the weights from the hidden to output layer.

v) The training/learning is very fast.

vi) The networks are very good at interpolation.

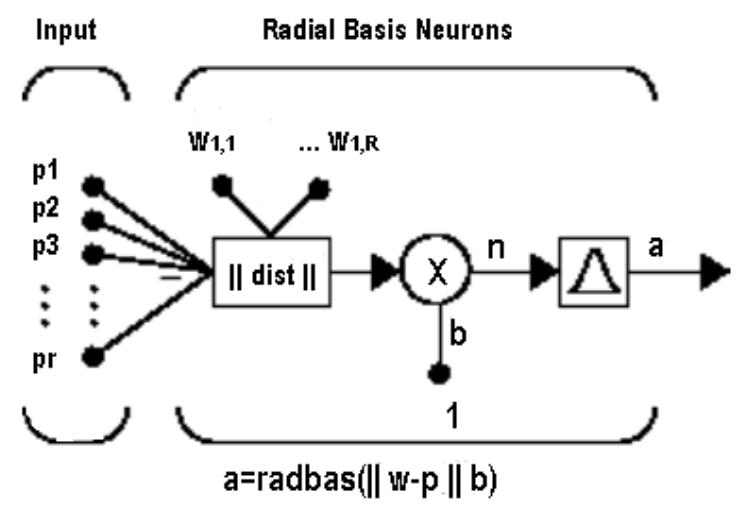

Fig3 : RBF Basis Structure

This system used the database of the Turkish license plates characters and some images from camera all 33 alphanumeric characters (23 alphabets and 10 numbers). The database formed is shown in Figure 7.

\section{ABCDEFGHIJKL} MNOPRSTUVYZ 0123456789

Fig4: The Database Character

Because of the similarities of some characters, there may be some errors during recognition. The confused characters mainly are I and 1, B and 8, E and F, D and O, S and 5, Z and 2.With these methods of radial basis function for recognition of characters and applied tests during recognition algorithm, recognition rate is improved with the minimum error.

Table II Experimental Results By Radial Basis Function

\begin{tabular}{|l|l|l|}
\hline $\begin{array}{l}\text { Units of LPR } \\
\text { system }\end{array}$ & $\begin{array}{l}\text { Number of } \\
\text { Accuracy }\end{array}$ & $\begin{array}{l}\text { Percentage of } \\
\text { Accuracy }\end{array}$ \\
\hline $\begin{array}{l}\text { Extraction of } \\
\text { Plate region }\end{array}$ & $230 / 250$ & $92.1 \%$ \\
\hline Segmentation & $221 / 250$ & $88.4 \%$ \\
\hline $\begin{array}{l}\text { Recognition of } \\
\text { Characters }\end{array}$ & $248 / 250$ & $99.2 \%$ \\
\hline
\end{tabular}

3) Recongnition Status:

Table III Recognition Status Of The Images

\begin{tabular}{|l|c|c|}
\hline $\begin{array}{l}\text { TESTED } \\
\text { IMAGES }\end{array}$ & BACKGROUND & $\begin{array}{c}\text { CORRECT } \\
\text { RECOGNITION }\end{array}$ \\
\hline AED -632 & $\begin{array}{c}\text { Yellow } \\
\text { Background }\end{array}$ & $\begin{array}{c}\text { Yes } \\
\text { AED-632 }\end{array}$ \\
\hline DL4CAG9557 & Day Light & $\begin{array}{c}\text { Yes } \\
\text { DL4CAG9557 }\end{array}$ \\
\hline DL2CAD0311 & Having angle & $\begin{array}{c}\text { Yes } \\
\text { DL2CAD0311 }\end{array}$ \\
\hline UP14CB7145 & White Background & $\begin{array}{c}\text { Yes } \\
\text { UP14CB7145 }\end{array}$ \\
\hline
\end{tabular}

\section{CONCLUSION}

In this paper, we presented application software designed for the recognition of car's license plate. In this firstly we extracted the plate location, then we separated the plate characters individually by segmentation and finally applied Feed Forward Network and Radial Basis Function and get the result that Radial Basis Function is better for the recognition of License plate. The proposed approach could be used with combination with other ones for better security and increasing the area of the particular application.

\section{REFERENCES}

[1] Muhammad H Dashtban, Zahra Dashtban"A Novel Approach for Vehicle License Plate Localization and Recognition" International Journal of Computer Applications (0975 - 8887) Volume 26- No.11, July 2011.

[2] A.Broumandnia, M.Fathy, (2005, Dec.), Application of pattern recognition for Farsi license plate recognition, to be presented at ICGST International Conference on Graphics, Vision and Image Processing (GVIP-05). [Online]. Available: http://www.icgst.com/gvip /v2/P1150439001.pdf.

[3] Anish Lazrus, Siddhartha Choubey, Sinha G.R. "An Efficient Method Of Vehicle Number Plate Detecttion And Recognition" International Journal of Machine Intelligence ISSN: 0975-2927 \& E-ISSN: 0975-9166.

[4] H. J. Kim, D. W. Kim, S. K. Kim, J. V. Lee, J. K. Lee, 1997, "Automatic Recognition of Car License Plates Using Colour Image Processing," Engineering Design \& Automation, 3(2),pp.215-225,1997. 
[5] M.M.M. Fahmy, 1994, "Automatic Number-plate Recognition : Neural Network Approach," Proceedings of VNIS'94 Vehicle Navigation and Information System Conference, 31 Aug-2 Sept, 1994.

[6] S.Kranti, K.Pranathi,A.Srisaila ," Automatic Number Plate Recognition" Information Technology ISSN 09764860

[7] Dheeraj Sinhmar, Ajay Mittal (2013). Vehicle Licence Plate Recognition Using Gaussian Hermite Moments and Wavelets. (pp. 404-409).
[8] Mahmood Ashoori-Lalimi, Sedigheh Ghofrani (2011). An Efficient Method for Vehicle License Plate Detection in Complex Scenes. (pp. 320-325).

[9] Divya Gilly, Kumudha Raimond (2013). A Survey on License Plate Recognition Systems. (pp. 0975-8887).

[10] M. Nilsson, J. Nordberg, I. Claesson. Face Detection using Local SMQT Features and SplitUp SNoW Classifier, IEEE International Conference on Acoustics, Speech, and Signal Processing(ICASSP),2007,2(4) ,pp: 589-592. The 2nd International Conference on Computer Application and System Modeling (2012). 